\title{
Reply to: Preventive role of ramelteon and suvorexant for postoperative delirium after pharyngolaryngectomy with esophagectomy
}

\author{
Eisuke Booka ${ }^{1,4,6} \cdot$ Yasuhiro Tsubosa $^{1} \cdot$ Teruaki Matsumoto $^{2} \cdot$ Mari Takeuchi $^{2,3} \cdot$ Takashi Kitani $^{4} \cdot$ Masato Nagaoka $^{4}$. \\ Atsushi Imai ${ }^{4} \cdot$ Tomoyuki Kamijo $^{4} \cdot$ Yoshiyuki lida $^{4} \cdot$ Ayako Shimada $^{1,4,6} \cdot$ Katsushi Takebayashi $^{1} \cdot$ Masahiro Niihara $^{1}$. \\ Keita Mori ${ }^{5} \cdot$ Tetsuro Onitsuka $^{4} \cdot$ Hiroya Takeuchi $^{6} \cdot$ Yuko Kitagawa $^{6}$
}

Received: 5 March 2018 / Accepted: 8 March 2018 / Published online: 18 April 2018

(c) The Japan Esophageal Society and Springer Japan KK, part of Springer Nature 2018

We would like to express our appreciation to Dr. Kawada for his attention and comments.

We totally agree with his comments. We also had the same concern as he expressed in his letter. However, we thought the results might interest the readers and might be of some use to those who were engaged in the management of postoperative delirium. Therefore, in spite of the incompleteness in statistical treatment, we thought it worth to report the results of this small research. We also think the results should be validated by randomized control trial procedure.

We reported the results of the multivariable logistic regression analysis based on only 9 events in 65 patients, because this study was conducted as an effort of our team therapy to prevent postoperative complications of esophagectomy and the objective was to understand the

This reply refers to the article available at https://doi.org/10.1007/ s10388-018-0603-2.

Yasuhiro Tsubosa

y.tsubosa@scchr.jp

1 Division of Esophageal Surgery, Shizuoka Cancer Center Hospital, 1007 Shimonagakubo, Nagaizumi-cho, Sunto-gun, Shizuoka 411-8777, Japan

2 Division of Psycho-Oncology, Shizuoka Cancer Center Hospital, Shizuoka, Japan

3 Palliative Care Center, Keio University Hospital, Tokyo, Japan

4 Division of Head and Neck Surgery, Shizuoka Cancer Center Hospital, Shizuoka, Japan

5 Clinical Trial Coordination Office, Shizuoka Cancer Center Hospital, Shizuoka, Japan

6 Department of Surgery, Keio University School of Medicine, Tokyo, Japan preventive effect of the combination of ramelteon and suvorexant to postoperative delirium using the available data. As pointed out by the letter, there is some incompleteness in the statistical treatment. Especially, based on the one in ten rule, we should have included more than 20 events in the analysis. We think that we should have displayed some statistical information such as confidence interval, sample size, event size and so on. We also should have included some statistical limitations in the article, so that the readers could interpret the results properly without confusion.

Regarding individual data, retrospective single institute researches may have some kind of biases, but all patients' charts were systematically assessed by two psychiatrists to minimize them.

Regarding the recommendation to include minor tranquilizer as one of the variables, we did it as shown in Table 2. This retrospective study led to the conclusion that ramelteon with or without suvorexant was more effective than minor tranquilizer.

We hope the above could be a good explanation to the comments in Dr. Kawada's letter. 